\title{
Topical Liposomal Ozonated Oil in Complicated Corneal Disease: A Report on Three Clinical Cases
}

Fedele Passidomo'

Francesco Pignatelli (D)

Giuseppe Addabbo'

Ciro Costagliola (iD ${ }^{2}$

'Comprehensive Ophthalmology Unit, S. S. Annunziata Hospital, Taranto, Italy; ${ }^{2}$ Department of Medicine and Health Sciences "V. Tiberio", University of Molise, Campobasso, Italy
Correspondence: Fedele Passidomo

Comprehensive Ophthalmology Unit, S.S.

Annunziata Hospital, Via Francesco

Bruno, I, Taranto, 74I2I, Italy

Tel +393288569l 25

Email dotpaoli@yahoo.it

\begin{abstract}
Infectious and traumatic corneal diseases require adequate therapy against infection and promote re-epithelialization. The appearance of multidrug-resistant bacterial strains, induced by excessive use of antibiotics in the treatment of infectious diseases, has urged researchers to study new antimicrobial formulations. Ozonated oils have demonstrated antiseptic (antibacterial, antifungal and antiviral) and reparative properties and can be proposed for the prevention and treatment of acute and chronic local infections, as an alternative to conventional topical antimicrobials. In ocular treatment, ozonated oil can exert tissue repair and anti-inflammatory and bactericidal activity. Moreover, its specific formulation makes it well tolerated and biocompatible with the ocular surface. Herein, we report on three cases of corneal inflammation (dendritic epithelial keratitis, corneal injuries caused by olive leaves, and neurotrophic corneal ulcer), treated with liposomal ozonated oil (Ozodrop $^{\circledR}$, FB VISION S.p.a. San Benedetto del Tronto, Italy), successfully resolved using topical liposomal ozonated oil.
\end{abstract}

Keywords: corneal trauma, corneal ulcers, corneal wound healing, infectious keratitis, ozonated oil

\section{Introduction}

Infectious and traumatic corneal diseases require adequate and multi-factorial therapy able to provide protection against infection and promote reepithelialization. Excessive use of antibiotics in the treatment of infectious diseases, use of inappropriate dosing regimens, and widespread agricultural and veterinary use of antibiotics have significantly contributed to the development of antibiotic resistance, ${ }^{1}$ an issue that has also been observed amongst the pathogens that cause infections specific to the ophthalmological field. ${ }^{2}$

Besides diligent surveillance for resistant bacteria, rigorous implementation of infection control practices, and restricted use of antibiotics for non-human and non-infectious purposes, another important strategy in countering the mounting threat of antibiotic resistance is the development of new antimicrobial agents with novel mechanisms of action. Of these, ozone is increasingly considered a treatment option for several types of infection. ${ }^{3}$ Ozone's antimicrobial, antifungal, and antiviral properties are well-known, as is its ability to prevent the development of antimicrobial resistance and allergic reactions. ${ }^{4}$ There has consequently been an increase in the use of ozonated oil in clinical practice for 
the treatment of a number of infectious and inflammatory conditions, including skin diseases, gum disease and diabetic foot ulcers.

Eye drops containing ozone were recently used in the management of ocular surface infection caused by bacteria, viruses and fungi, ${ }^{4-6}$ and a specific formulation containing liposomal ozonated sunflower oil (Ozodrop ${ }^{\circledR}$, FB Vision, San Benedetto del Tronto, Italy) that is well tolerated by the conjunctival mucosa and ocular surface has been developed. ${ }^{7}$

The most frequently diagnosed anterior segment conditions are uveitis, corneal abrasion, corneal ulcer, Meibomian gland dysfunction, dry eye, blepharitis, punctate epithelial erosions, conjunctivitis and epidemic keratoconjunctivitis. Herein we report on three cases of corneal inflammation (dendritic epithelial keratitis, corneal injuries caused by olive leaves, and neurotrophic corneal ulcer) treated with liposomal ozonated oil. All three patients gave their written informed consent to participate in this case report after receiving a detailed description of the procedure to be used and the purpose of the study, and their written agreement to publish case reports.

\section{Case I - Dendritic Epithelial Keratitis}

A 56-year-old man came to our Ophthalmology Unit complaining of blurred vision, foreign body sensation, burning and itching in his right eye. His best corrected visual acuity (BCVA) was $5 / 10$ in the right eye. The patient did not wear corrective spectacles, and denied trauma and exposure to chemicals.

The slit lamp examination revealed slight lid oedema and conjunctival hyperaemia; and the fluorescein stain test revealed branching epithelial lesions with terminal bulbs consistent with the situation that is classically observed with herpes simplex virus (HSV) epithelial keratitis (shown in Figure 1A). The corneal stroma was clear, and there was no inflammation in the anterior chamber or posterior segment. The patient's left eye was normal. Valaciclovir $1000 \mathrm{mg}$ tablets ( 1 tablet/day) and liposomal ozonated oil eye drops 3 times a day in the right eye were prescribed. Three days later, a decrease in the fluorescent area and dendritic thickness (Figure 1B), accompanied by a reduction in pain, lacrimation and photophobia was observed. By day four, the corneal involvement and fluorescein staining were practically resolved (Figure 1C), and a further reduction in symptoms occurred. After one week of treatment, the corneal examination showed good healing of the defect (Figure 1D), and a full recovery of visual acuity in right eye was achieved.

In this clinical case, adding liposomal ozonated oil to the systemic antiviral therapy significantly shortened the healing time and reduced the clinical symptoms in just 7 days. After the first week, the patient suspended the systemic antiviral treatment, and continued liposomal ozonated oil (3 times a day) alone for 1 month, leading to complete recovery of sight, no signs of corneal opacities and a complete regression of the symptoms.

\section{Case 2 - Corneal Trauma Caused by Olive Leaves}

A 65-year-old farmer came to our first aid service for severe pain, intense lacrimation and a foreign body sensation. The patient's symptoms began after an olive leaf accidentally injured his right eye while harvesting olives. At admission, his BCVA was 4/10 in the right eye and 10/10 in the left eye. The slit lamp examination revealed upper lid oedema, conjunctival redness, and a large corneal abrasion involving the nasal and lower-nasal zones of the cornea (from 2 to 6 o'clock on the fluorescein eye stain test) (Figure 2A).
A

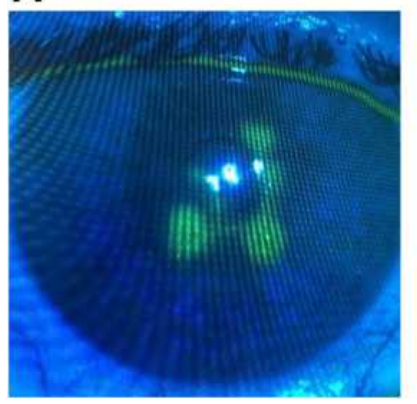

B

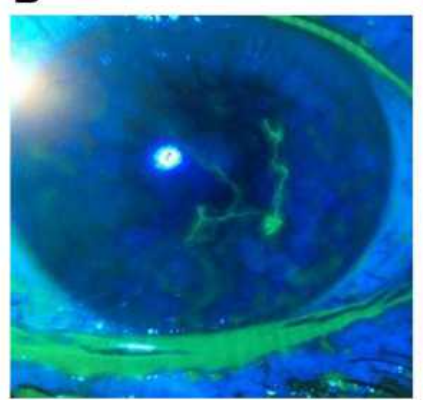

C

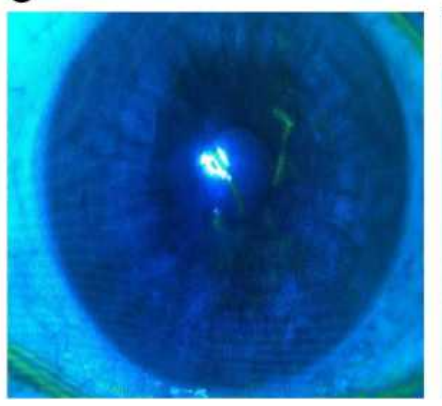

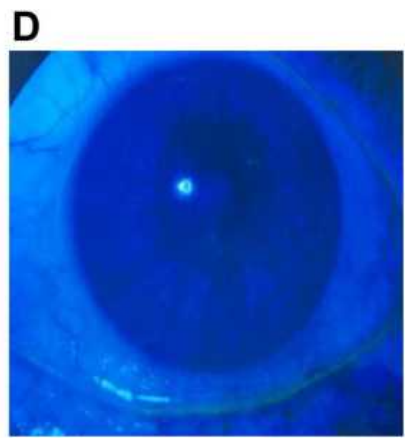

Figure I Right eye of a 56-year-old patient with dendritic epithelial keratitis during the follow-up period ((A): baseline; (B): after 3 days; (C): after 4 days; (D): after 7 days). Notes: The rapid resolution of the viral keratitis after systemic antiviral therapy and topical treatment with liposomal ozonated oil is evident throughout the follow-up period. 

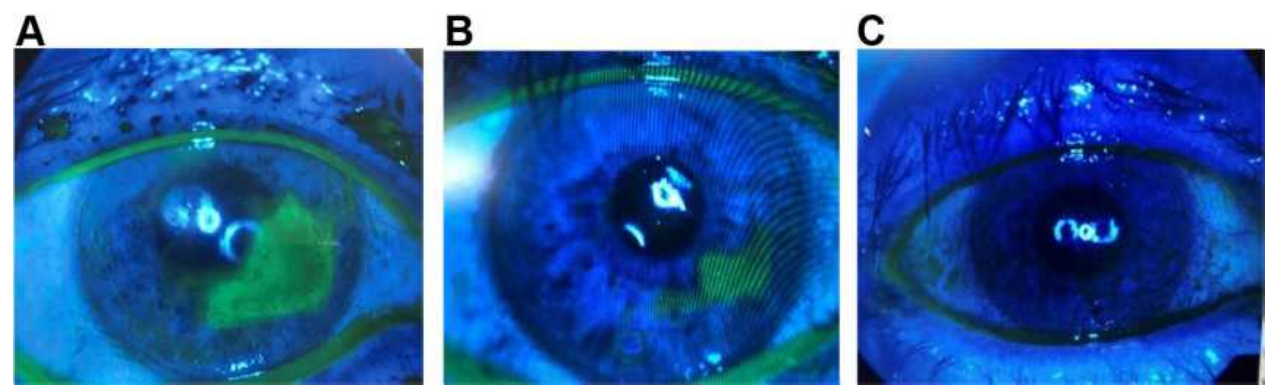

Figure 2 A 65-year-old man immediately after a corneal trauma caused by an olive leaf ((A): Large inferior trauma between 2 and 6 o'clock; (B): after 2 days; (C): after 3 days of treatment).

Liposomal ozonated oil eye drops, 6 times a day, plus artificial tears 3 times a day, and an eye bandage were prescribed. Two days later, the abrasion had shrunk by about $50 \%$ (Figure 2B), with a partial resolution of the sub-oedema, and a reduction in corneal staining. The foreign body sensation and lacrimation had diminished. BCVA had improved to $6 / 10$. Three days later, the corneal epithelium had healed completely (Figure 2C), BCVA had reached 10/10, and the symptoms had completely resolved. The therapy was continued for a further month using the same regimen, and no signs of corneal opacities were observed.

\section{Case 3 - Neurotrophic Corneal Ulcer}

A 63-year-old woman was hospitalised for a corneal abscess. Three days earlier, due to severe redness and a foreign body sensation, the patient had received topical antibiotic therapy with a rapid improvement in the objective clinical signs and symptoms, which subsequently got worse. At admission, the patient presented lagophthalmos, associated with the inability to close her left eyelid completely (Bell's palsy), presence of conjunctival hyperaemia, with an accumulation of mucous and purulent secretions, and persistent discharge at the fornices. She also presented severe corneal infection, corneal abscess, and corneal oedema with Descemet's folds (Figure 3A). Anterior segment OCT images revealed hyper-reflectivity with high scattering effect due to a dense accumulation of abscess material and an increased corneal thickness (Figure 3B). Conjunctival swabs were taken for microbiological investigations, and the patient started therapy with Ceftazidime pentahydrate for injection, $2 \mathrm{~g} / \mathrm{vial}$ (1 vial/ day), Atropine $1 \%$ eye drops (2/day), Levofloxacin eye drops (hourly), Gentamicin eye drops (hourly), and liposomal ozonated oil eye drops (5/day). After 3 days of treatment, the objective clinical situation showed an initial improvement, with a reduction of abscess area, corneal oedema and corneal infection (Figure 3C). After 5 days, a further improvement was seen, and initial draining of the abscess was performed (Figure 3D).

Systemic therapy with Acyclovir $400 \mathrm{mg}$ tablets, 5 times a day for 5 days was prescribed. Corneal sensitivity was qualitatively evaluated with a wisp of the cottontipped applicator; complete anaesthesia was observed in all 4 quadrants of the cornea. The presence of palsy, lagophthalmos, inability to close the left eyelid completely, eye pain and the corneal patterns at confocal microscopy suggested the corneal abscess had a neurotrophic aetiology. Acyclovir was continued at $400 \mathrm{mg}$ twice-daily for 15 days, then $400 \mathrm{mg}$ /day for 1 month. The confocal microscopy revealed the presence of severe inflammatory cell infiltrate, which almost completely replaced the keratocytes, caused by the viral agent (Figure 3E). Clusters of round inflammatory cells and corneal nerve fibre damage were evident, as neurotrophic keratopathy can exacerbate autoimmune post-herpetic stromal keratitis.

Therapy with liposomal ozonated oil (3 times a day) was continued, and after 7 days, a significant reduction was seen in the affected area on the fluorescein test (Figure $3 \mathrm{~F})$. Ten days later, restoration of epithelial integrity was observed (Figure $3 \mathrm{G}$ and $\mathrm{H}$ ) with a slight positive fluorescein test. The OCT showed a gradual and progressive normalisation of corneal anatomy, with resolution of the abscess area and ulcer (Figure 3I, L and M).

\section{Discussion}

Corneal inflammation represents a challenge in current ophthalmological practice, due to its diverse aetiologies and the risk of infection and superinfection. The appearance of resistant and multi-resistant bacterial strains, such as methicillin-resistant Staphylococcus aureus and 
A
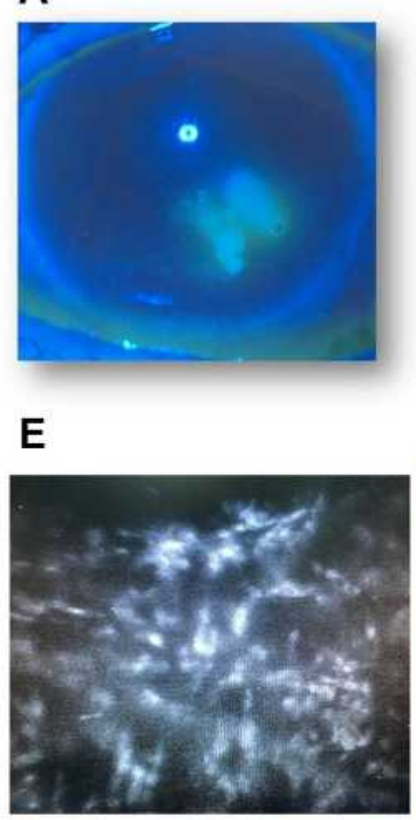

$\mathbf{F}$

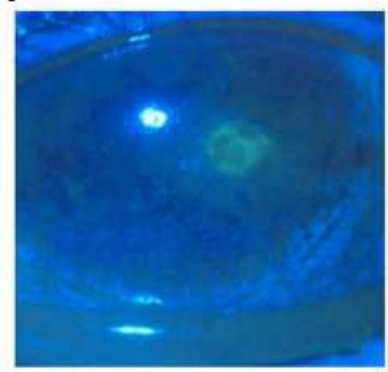

C
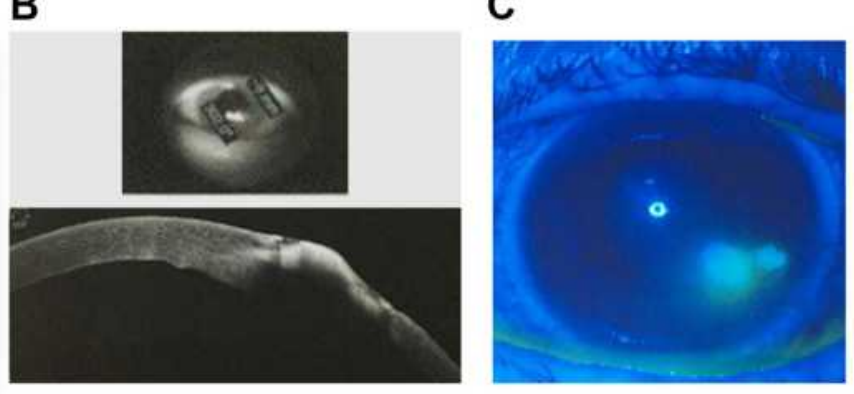

G

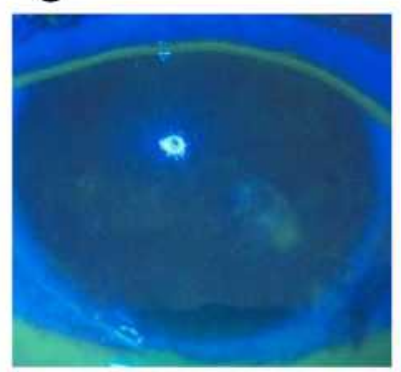

D

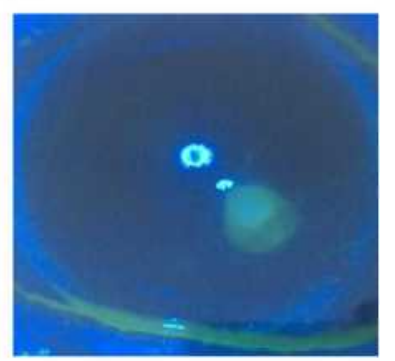

H

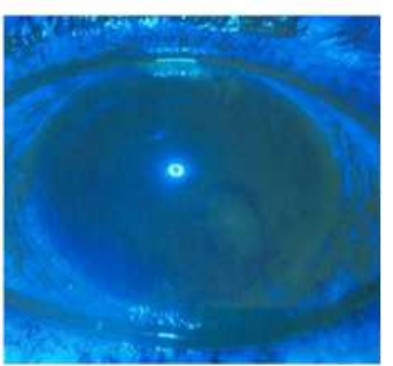

I

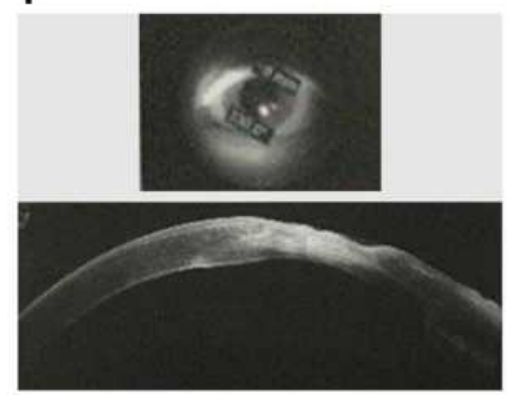

J

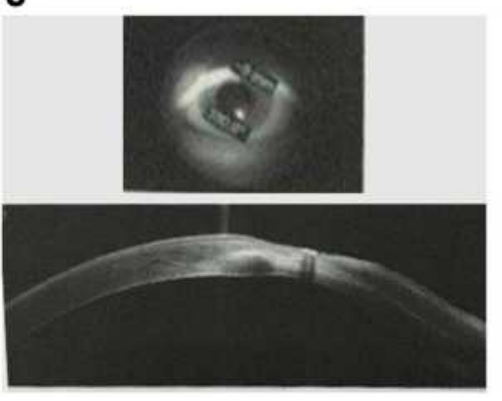

K

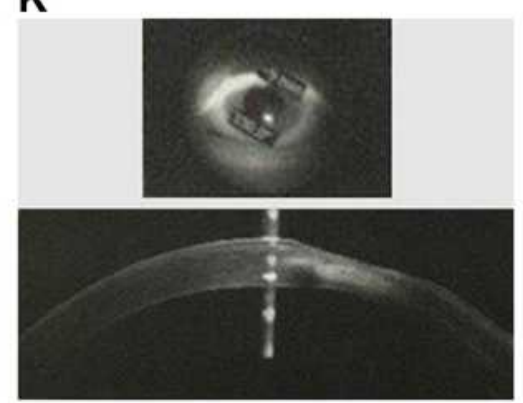

Figure 3 Corneal abscess in a 63-year-old woman ((A): slit lamp; (B): corneal OCT; (C): after 3 days; (D): after 5 days; (E): corneal confocal microscopy after 5 days; (F): corneal abscess nearly solved after 7 days; $(\mathbf{G})$ : after 10 days; $(\mathbf{H})$ : after 12 days; $(\mathbf{I}-\mathbf{K})$ : the OCT examination shows a gradual and progressive normalisation of corneal anatomy, with resolution of the abscess area and the ulcer).

Pseudomonas aeruginosa, has urged researchers to investigate new antimicrobials able to cover a broad spectrum of microorganisms. Of these, liposomal ozonated oil has shown antiseptic and reparative properties and can be proposed for the prevention and treatment of acute and chronic local infections, as an alternative to topical antimicrobial agents, on account of its antibacterial, antifungal and antiviral properties. ${ }^{4-6}$ Liposomal ozonated oil can therefore be a useful alternative for countering the widespread and indiscriminate use of antibiotics, which has led to the emergence of bacterial resistance, especially in Staphylococci sp. Moreover, topical ozonated oil was seen to accelerate acute cutaneous wound repair in Guinea pig models by promoting collagen synthesis and fibroblast proliferation at the injury site. The topical form of $\mathrm{O}_{3}$ increases the expression of growth factors, such as
PDGF, TGF- $\beta$, and VEGF, and by activating transcription factor NF- $\mathrm{KB}$ it is able to regulate inflammatory responses and eventually the entire wound-healing process. ${ }^{8}$

The Comprehensive Ophthalmology Unit of Ospedale SS Annunziata in Taranto, Italy, operates in a high-risk area, due to the presence of a large steel industry and extensive agricultural activities. More than 10,000 eye injuries caused by accidental contact with ferrous materials and metallic foreign bodies, as well as with sharp leaves, tree trunks or stinging plants are examined each year. All these clinical conditions present a high risk for bacterial, mycotic or viral infections.

Herpetic keratitis is the most prevalent infectious cause of corneal blindness in developed countries. Approximately 10 million people worldwide may have herpetic eye disease and the primary infection may occur 
at any time in life. The dendritic form of keratitis presents the most frequent recurrences (56.3\%), whereas the stromal and geographical forms are less common (29.5\% and $9.8 \%$, respectively). ${ }^{9}$ The mainstay of viral keratitis therapy is antiviral treatment either in the form of oral Valacyclovir for 10 to 14 days or topical antiviral medications (Acyclovir, Ganciclovir), usually for 2 weeks. ${ }^{10}$ Topical application often results in superficial punctate keratitis, chemical conjunctivitis, punctal occlusion, and rare hypersensitivity reactions due to corneal epithelial defects, which affects the normal wound-healing process. ${ }^{11}$ In our experience, adding novel liposomal ozonated oil to systemic antiviral therapy made it possible to avoid bacterial superinfection without using antibiotic eye-drops, and significantly shortened the healing time and clinical symptoms. The preparation has been effective in improving healing times, resolving symptoms, and protecting the ocular surface from infection, either in monotherapy or as adjunctive treatment.

According to AAO, oral antiviral agents appear to be as effective as topical antiviral agents in the treatment of HSV epithelial keratitis. There is no evidence that simultaneous use of two antiviral agents, whether topical or oral, accelerates healing of HSV epithelial keratitis. ${ }^{12}$ For this reason, we have preferred a systemic antiviral approach (patient had not a renal impairment) to avoid antiviral ointment (less comfortable for the patient) and benzalkonium chloride (present in acyclovir topical formulation), and to exploit topical ozone formulation antiviral and anti-inflammatory potential, and its fast healing property.

Eye trauma accounts for about 3\% of all emergency department admission and approximately $80 \%$ of these visits are for corneal abrasions or foreign bodies. Contact lens wear, or fingernails, plant branches or leaves that accidentally meet the ocular surface may cause corneal abrasion, whose incidence is highest in the working age population. As most corneal abrasions heal spontaneously, treatment consists primarily in pain control and prevention of infection. ${ }^{13}$ Ozone is highly effective on bacteria, included multiresistant, because it blocks the enzymatic function on bacteria by oxidizing glycoproteins and glycolipids. To enter inside the bacterial cell, ozone oxidizes the phospholipids and lipoproteins of the bacterial cell envelope, disrupting the cytosolic membrane integrity. Owing to its high wound healing capacity, its influence on the processes of oxygen metabolism, and antibacterial properties, ozone has been used as a complementary treatment in infected diabetic foot ulcers, impairing the healing and reducing the infection and amputation rates. ${ }^{14}$

Furthermore, ozonated oil has a high capacity to remove bacterial biofilms; this, together with the action on planktonic bacterial cells, and its described safety features make ozonated oil a promising alternative to the current antibiotics and disinfectants ${ }^{14}$ also in ophthalmology, allowing in selected cases to avoid antibiotics or enlarging their spectrum of action to other microbes (eg fungi and viruses) or to multiresistant bacteria (eg MRSA) protecting the cornea from possible superinfection, too.

Neurotrophic keratitis (NK) is a degenerative corneal disease characterised by a partial or total loss of corneal sensitivity. In NK, corneal innervation by the trigeminal nerve is impaired. Partial or complete loss of corneal sensitivity may result in epithelial keratopathy, epithelial defects, stromal ulceration, and eventually corneal perforation. Its epidemiology is still unclear. As a rare disease, it has an estimated prevalence of less than 50/ 100,000 individuals. Any ocular or systemic condition able to affect corneal sensory innervation can result in NK. ${ }^{15}$

Many factors may have contributed to the development of the corneal abscess; lagophthalmos, inability to completely close the left eyelid, and chronic dryness due to palsy, are all contributing factors for corneal ulcer. Furthermore, in this case, a herpetic infection exacerbated by an already compromised anatomical substrate.

Adjunctive therapy with liposomal ozonated oil played an important role in promoting wound healing and acted as an antiseptic. Due to its reparative effect, liposomal ozonated oil can support standard therapy by promoting faster healing and a reduction in symptoms, as well as protecting the cornea against infection, thanks to its broad spectrum of action. In fact, the reduction in the microbial load at the lesion site promotes corneal wound healing, without the risk of opacity or irregular repair, resulting in an even ocular surface.

These selected clinical cases, although anecdotic, demonstrate that liposomal ozonated eye drops represent useful adjunctive therapy for the management of both infectious and traumatic anterior segment conditions, in that they expand the antibiotic spectrum, eliminate the risk 
for potentially resistant bacteria, whilst acting on fungi and viruses, and significantly shorten healing times and the duration of clinical symptoms.

\section{Statement of Ethics}

Institutional Review Board of S.S. Annunziata Hospital did not require an approval to publish the case details. Each subject gave his/her written informed consent to publish his/her case and related images. Clinical cases are performed using a certified product, and not an experimental one, under the approved indications Ozonized oil in liposomes is indicated in case of inflammatory diseases of the cornea (keratitis) and conjunctiva (conjunctivitis), including those of infectious origin (from bacteria, viruses, fungi) and/or in case of abrasions, corneal trauma, use of contact lenses.

\section{Author Contributions}

All authors made a significant contribution to the work reported, whether that is in the conception, study design, execution, acquisition of data, analysis and interpretation, or in all these areas; took part in drafting, revising or critically reviewing the article; gave final approval of the version to be published; have agreed on the journal to which the article has been submitted; and agree to be accountable for all aspects of the work.

\section{Funding}

The publishing fee was covered by FB VISION.

\section{Disclosure}

The authors report no conflicts of interest in this work.

\section{References}

1. Ventola CL. The antibiotic resistance crisis: part 1: causes and threats. PT. 2015;40:277-283.
2. Ting DSJ, Ho CS, Cairns J, et al. 12-year analysis of incidence, microbiological profiles and in vitro antimicrobial susceptibility of infectious keratitis: the Nottingham Infectious Keratitis Study. $\mathrm{Br}$ J Ophthalmol. 2020.

3. Smith NL, Wilson AL, Gandhi J, Vatsia S, Khan SA. Ozone therapy: an overview of pharmacodynamics, current research, and clinical utility. Med Gas Res. 2017;7(3):212-219. doi:10.4103/2045-9912. 215752

4. Celenza G, Iorio R, Cracchiolo S, et al. Antimycotic activity of ozonized oil in liposome eye drops against Candida spp. Transl Vis Sci Technol. 2020;9:4. doi:10.1167/tvst.9.8.4

5. Spadea L, Tonti E, Spaterna A, Marchegiani A. Use of ozone-based eye drops: a series of cases in veterinary and human spontaneous ocular pathologies. Case Rep Ophthalmol. 2018;9:287-298. doi:10. $1159 / 000488846$

6. Cagini C, Mariniello M, Messina M, et al. The role of ozonized oil and a combination of tobramycin/dexamethasone eye drops in the treatment of viral conjunctivitis: a randomized clinical trial. Int Ophthalmol. 2020;40:3209-3215. doi:10.1007/s10792-020-015 03-4

7. Mohamed MSM, Abdallah AA, Mahran MH, Shalaby AM. Potential alternative treatment of ocular bacterial infections by oil derived from Syzygium aromaticum flower (clove). Curr Eye Res. 2018; 43:873-881. doi:10.1080/02713683.2018.1461907

8. Kim HS, Noh SU, Han YW, et al. Therapeutic effects of topical application of ozone on acute cutaneous wound healing. J Korean Med Sci. 2009;24:368-374. doi:10.3346/jkms.2009.24.3.368

9. Farooq AV, Shukla D. Herpes simplex epithelial and stromal keratitis: an epidemiologic update. Surv Ophthalmol. 2012;57(5):448-462. doi:10.1016/j.survophthal.2012.01.005

10. Sibley D, Larkin DFP. Update on Herpes simplex keratitis management. Eye (Lond). 2020;34:2219-2226. doi:10.1038/s41433020-01153-x

11. Raizman MB, Hamrah P, Holland EJ, et al. Drug-induced corneal epithelial changes. Surv Ophthalmol. 2017;62:286-301. doi:10.1016/ j.survophthal.2016.11.008

12. White ML, Chodosh J. Herpes simplex virus keratitis: a treatment guidelines. Am Acad Ophthalmol. 2014.

13. Willmann D, Fu L, Melanson SW. Corneal injury. In: StatPearls [Internet]. Treasure Island (FL): StatPearls Publishing; 2020.

14. Silva V, Peirone C, Amaral JS, et al. High efficacy of ozonated oils on the removal of biofilms produced by methicillin-resistant Staphylococcus aureus (MRSA) from infected diabetic foot ulcers. Molecules. 2020;25(16):3601. doi:10.3390/molecules25163601

15. Versura P, Giannaccare G, Pellegrini M, Sebastiani S, Campos EC. Neurotrophic keratitis: current challenges and future prospects. Eye Brain. 2018;10:37-45. doi:10.2147/EB.S117261
International Medical Case Reports Journal

\section{Publish your work in this journal}

The International Medical Case Reports Journal is an international, peer-reviewed open-access journal publishing original case reports from all medical specialties. Previously unpublished medical posters are also accepted relating to any area of clinical or preclinical science. Submissions should not normally exceed 2,000 words or 4 published pages including figures, diagrams and references. The manuscript management system is completely online and includes a very quick and fair peer-review system, which is all easy to use. Visit http://www.dovepress.com/testimonials.php to read real quotes from published authors. 